\title{
Turismo accesible y evaluación de usuarios con diversidad funcional visual. Los proyectos OPERA y PRA2
}

\section{Accessible tourism and evaluation of user's with visual disability. The OPERA and PRA2 projects}

\section{Palabras clave}

Traducción, accesibilidad, audiodescripción, estudios de recepción, evaluación.

\section{Keywords}

Translation, accessibility, audio description, reception studies, evaluation.

\section{Cristina Álvarez de Morales Mercado \\ <cristinaalvarez@ugr.es>}

Universidad de Granada. España

\section{Introducción ${ }^{1}$}

El artículo que presentamos se enmarca dentro de I+D Acceso al ocio y la cultura. Plataforma de difusión y evaluación de recursos audiovisuales accesibles OPERA, concedido en 2016 y que se está desarrollando en la actualidad. El proyecto OPERA nace con un objetivo fundamental: perfeccionar e impulsar la plataforma PRA2, desarrollada en un proyecto I+D anterior y que consiste en un sitio web de consulta y evaluación en línea de recursos audiovisuales accesibles, a través de la traducción y la interpretación para personas con discapacidad sensorial. Si en el proyecto anterior el principal ámbito de investigación fue el de los medios de comunicación audiovisuales, en este caso, el proyecto OPERA se centra en el turismo y la museografía accesibles y pretende alcanzar los siguientes objetivos:

1. Evaluar los recursos de accesibilidad existentes y los de nueva creación.

2. Proporcionar visibilidad a los recursos accesibles así como a las empresas y a los profesionales responsables de la creación de ellos.

1. Este artículo ha sido financiado por el I+D OPERA. Acceso al ocio y la cultura. Plataforma de difusión y evaluación de recursos audiovisuales accesibles (Código: FFI2015-65934-R).

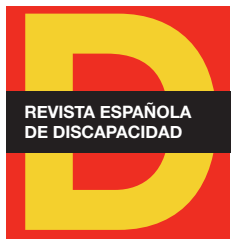

Para citar:

Álvarez de Morales, C. (2018):

"Turismo accesible y evaluación de usuarios con diversidad funcional visual. Los proyectos OPERA y PRA2". Revista Española de Discapacidad, 6 (I): 305-316.

Doi: <https://doi.org/10.5569/23405104.06.01.16> 
3. Posibilitar la transferencia del conocimiento entre la investigación universitaria, por un lado, y las empresas y asociaciones de usuarios, por otro, por medio de informes de resultados de los estudios llevados a cabo a través de la plataforma.

La plataforma, totalmente accesible, fomenta la comunicación entre el conjunto formado por instituciones culturales, empresas privadas de difusión del arte y la cultura y los proveedores de servicios de asesoramiento y desarrollo en materia de accesibilidad al ocio y la cultura.

Figura 1. Plataforma PRA2

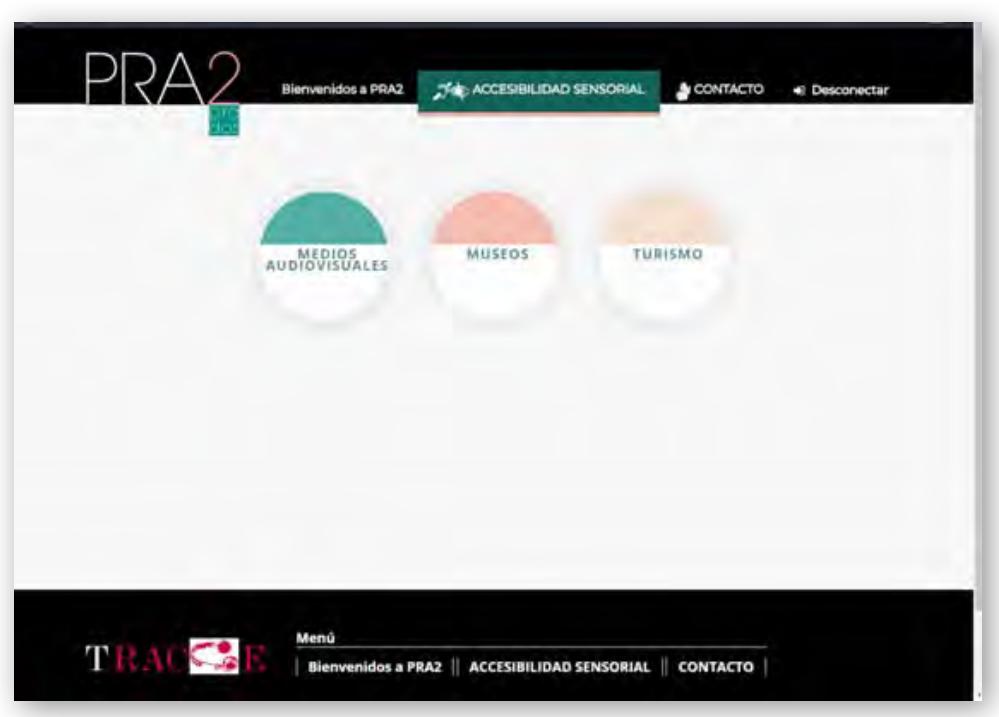

Fuente: elaboración propia.

El impacto social de estos objetivos es conseguir una mayor difusión de los principios de la accesibilidad universal que repercuta en las instituciones y empresas de difusión de la cultura y el conocimiento y conlleve un aumento considerable de las medidas destinadas a mejorar el acceso a sus productos y servicios por parte de las personas con discapacidad.

\section{Estado de la cuestión}

La plataforma de recursos audiovisuales accesibles PRA2 (https://tracce.ugr.es/pra2/) contempla las modalidades de traducción e interpretación de la audiodescripción (AD), el subtitulado para personas sordas (SpS) y la interpretación en lengua de signos (ILS), y se compone de dos secciones fundamentales: recursos audiovisuales accesibles y estudios de recepción de dichos recursos. Los recursos están agrupados en las categorías de medios audiovisuales (Pettitt et al., 1996), turismo y museos (Jiménez et al., 2012; Soler y Chica, 2014). 
Los estudios de recepción consisten en cuestionarios en línea que permiten la evaluación del recurso de accesibilidad por parte del usuario y hasta ahora se han centrado en la audiodescripción (Mazur y Kruger, 2012; Navarrete, 1997; Peli et al., 1996) y el subtitulado para personas sordas (Fryer y Freeman, 2012; Iglesias et al., 2015). Estos estudios se han diseñado con el fin de evaluar los métodos empleados, los recogidos por las directrices existentes en esta materia (AENOR 2005 y 2012) y métodos alternativos. Entre las instituciones que colaboran con el grupo TRACCE se encuentran el Museo Sorolla, el Museo Lázaro Galdiano, el Museo Julio Romero de Torres, el Ayuntamiento de Córdoba (como gestor de la accesibilidad en los monumentos del Real Alcázar de los Reyes Cristianos y los Baños del Alcázar Califal) y el Museo CajaGranada Memoria de Andalucía.

La evaluación de la calidad de los recursos de accesibilidad ha sido una prioridad desde los comienzos de la implantación de servicios de audiodescripción, subtitulado para personas sordas e interpretación en lengua de signos en la cultura y los medios de comunicación. No obstante, estos estudios se han centrado fundamentalmente en la audiodescripción y el subtitulado para personas sordas en el cine y la televisión. Sin embargo, son muy escasas aún las investigaciones que analizan la recepción de la audiodescripción en los ámbitos turístico y museográfico. Aquí cabe destacar la experiencia del grupo de investigación HUM770: Aula de investigación del texto multimedia, integrado por investigadores del proyecto TRACCE que han llevado a cabo múltiples proyectos ${ }^{2}$ con una vertiente de investigación aplicada en torno a la accesibilidad en museos y en el turismo para personas con discapacidad sensorial.

La principal innovación de la plataforma PRA2 es que permite llegar a un número mayor de usuarios gracias a que los cuestionarios se administran a través de una plataforma en línea. El método empleado en el diseño de los cuestionarios contempla dos tipos de procedimiento: (1) evaluación de material existente, y (2) manipulación de las variables. En el primer caso, se extraen, de uno o varios recursos existentes, fragmentos relacionados con una variable dependiente particular (traducción de las emociones, traducción del color, segmentación de subtítulos, léxico especializado en lengua de signos, etc.). En el segundo, se sigue asimismo el procedimiento anterior y seguidamente se manipula la variable estudiada para modificarla en el sentido de aplicar una alternativa lingüística, textual, traductológica o técnica. Para la manipulación de estos segmentos se emplean aplicaciones informáticas de audiodescripción, subtitulado y edición de vídeo no lineal.

En lo que respecta a su función como recurso de referencia de material cultural accesible, la plataforma PRA2 no solo facilita el proceso de búsqueda de recursos accesibles por parte de los usuarios, sino que, además, la inclusión de muestras de estos recursos permite al usuario comprobar la utilidad y adecuación de los mismos a sus necesidades antes de dirigirse a la institución cultural. También constituye una base de datos de proveedores, productos y clientes. 


\section{Estudio de dos casos}

\subsection{Materiales accesibles}

Para explicar mejor lo expuesto en el apartado anterior hemos seleccionado dos ejemplos de materiales audiodescritos que fueron evaluados por cuatro sujetos ciegos en una vista que el grupo de investigación TRACCE realizó a la sede de la ONCE de Granada para evaluar parte de los materiales accesibles elaborados para la plataforma: el primero, la fachada del Ayuntamiento de Pamplona y, el segundo, el Jardín Bajo del Alcázar de los Reyes Cristianos en Córdoba. Se han escogido estos dos elementos porque son ejemplos muy claros de los materiales utilizados por OPERA para su evaluación por usuarios con diversidad funcional visual. Ambos responden a la especialidad de turismo accesible por el que nos decantamos en este nuevo proyecto.

\section{- Ejemplo 1. Fachada del Ayuntamiento de Pamplona}

a. Documentación

El maestro José Zay y Lorda concibió una noble fachada de sillar, bien proporcionada y rica en matices barrocos, en la que a primera vista contrasta lo rectilíneo del paramento con el juego de columnas pareadas, exentas, que introducen un suave movimiento en el frontis. En alzado desarrolla tres cuerpos, más ático, cuyos extremos están enmarcados por un frontón curvo partido, en contraposición al triangular que remata el cuerpo del reloj. Muy clara resulta la lectura horizontal y vertical de esta estructura, cuyos pisos se separan por medio de la balconada, corrida en el central e individual en el tercero, más un entablamento con triglifos y metopas en el primero y ménsulas en el segundo. En contraposición el paso al ático viene dado por una balaustrada de piedra.

La estructuración vertical en tres calles con vanos rectos la ordenan dobles columnas exentas, que apoyan en un alto basamento con recuadramientos y que según los pisos varían el tratamiento del fuste, liso o acanalado, con el tercio inferior estriado o decorado, superponiéndose también los órdenes, dórico, jónico y compuesto. A este juego arquitectónico de gran riqueza hay que añadirle un amplio repertorio ornamental de hojarasca, temas geométricos, espejos con rocalla, etc., que realzan el enmarque de los vanos. Perfectamente meditado está el diseño de los mismos, todos rectos a excepción de la entrada, ennoblecida con un medio punto cajeado.

b. Material audiodescriptivo

"La fachada, que se nos presenta al fondo de la plaza, tiene forma rectangular y es en su mayoría de estilo barroco. Debido a los adornos y a su forma arquitectónica, hay quien no ha dudado en compararla con un reloj de pared muy tallado.

La portada está construida en tres pisos horizontales más un ático. Pero no sólo eso, si no que, en sentido vertical se divide en tres calles separadas por dobles columnas de estilos clásicos, así que el edificio que aparece ante nosotros nos recuerda en cierta manera a un mueble de cajones hecho en piedra gris.

La base del edificio está rematada por dobles columnas de estilo dórico, apoyadas en un basamento, que son las más firmes y robustas de entre los órdenes clásicos. En medio del piso bajo se abre 
la entrada con un arco de medio punto sobre el que está escrito en latín "La puerta está abierta para todos, pero sobre todo el corazón". Flanqueándola, sobre pedestales, hay dos figuras femeninas. A la derecha, con aspecto heroico como si de una Juana de Arco se tratase, tenemos la alegoría de la Justicia, que espada en mano, sujeta también una balanza en su izquierda"3.

\section{- Ejemplo 2. Jardín Bajo del Alcázar de los Reyes Cristianos en Córdoba}

\section{a. Documentación}

El Alcázar de los Reyes Cristianos, fortaleza y palacio de sólidos muros, encierra en su interior gran parte de la evolución arquitectónica de Córdoba. Restos romanos y visigodos conviven con los de origen árabe en este majestuoso solar, ya que fue lugar predilecto de los distintos gobernantes de la ciudad. Cuando en 1236 Córdoba es conquistada por Fernando III el Santo, el edificio, que formaba parte del antiguo Palacio Califal, estaba totalmente asolado. Alfonso X el Sabio comienza su restauración, completada durante el reinado de Alfonso XI. A lo largo de la historia se le ha dado múltiples usos, como sede del Santo Oficio (Inquisición), o cárcel (en la primera mitad del XIX). EI visitante que por primera vez vislumbra esta fortaleza se sorprende ante una construcción casi rectangular con extensos muros de sillares pétreos y cuatro torres que perfilan los ángulos (la de Los Leones, la del Homenaje, la de La Inquisición y la de Las Palomas) Dentro, las distintas dependencias se articulan en torno a patios con exóticas y bellas flores, hierbas aromáticas y frondosos árboles. Las estancias y corredores se cierran con cúpulas góticas de piedra4.

b. Material audiodescriptivo

"Esta parte del jardín, es la de mayor superficie y su traza se puede considerar prácticamente ortogonal. Queda configurada a mediados del siglo XX sobre una parte de la antigua Huerta del Alcázar. La comunicación entre el castillo y los jardines inferiores se hace a través de una serie de estanques a los que se accede bajando varios tramos de escalones de ladrillo. En este espacio nos encontramos con una fuente de piedra, que distribuye el agua a través de una acequia que pasa a unas albercas y unos pilones que sirven para regar estos jardines situados en la parte más occidental.

A lo largo del recorrido encontramos restos arqueológicos de fustes, capiteles, estatuas, así como otros restos arquitectónicos. De especial interés, son los espacios configurados para los dos trazados de estanques, con surtidores y parterres de flores de colores vivos, las alineaciones de naranjos a ambos lados junto a las albercas y la línea de cipreses modelados, todo lo cual, confiere a este jardín un carácter de reinterpretación del clásico modelo Hispano - Árabe"5.

\subsection{Encuesta}

El diseño de la encuesta constó de dos fases: (1) sondeo preliminar (estudio diagnóstico), en el que la encuesta provisional fue sometida a revisión por parte de los investigadores que formaban parte del proyecto OPERA, y (2) encuesta final, en la que se perfiló la versión definitiva de la encuesta.

3. Texto extraído de: <http://www.accesibilidaduniversal.org/proyectos/viendo-navarra/31-fachada-ayuntamiento>

4. Para más información: <http://www.turismodecordoba.org/seccion/alcazar-de-los-reyes-cristianos-de-cordoba>.

5. Texto extraído de: <https://alcazardelosreyescristianos.cordoba.es/?id=683\&ad=true>. 
La encuesta consta de cuatro preguntas de respuesta cerrada, que facilitan información sobre la identificación y perfil sociodemográfico del visitante (edad, sexo, profesión y formación), y otras seis preguntas generales de respuesta cerrada y abierta que se agrupan en varias categorías dependiendo de la clase y grado de discapacidad del visitante, sus hábitos de visitas turísticas y sus hábitos de uso de dispositivos móviles y tecnologías accesibles.

\subsection{Procedimiento}

El procedimiento seguido fue el siguiente: se citó a los participantes en una sala de la sede de la ONCE de Granada situada en la Plaza del Ayuntamiento de Granada. Todos los participantes, un total de 13 colaboraron activamente en la evaluación de algunos de los materiales seleccionados. Los investigadores se organizaron a su vez por grupos de trabajo y cada uno atendió a cuatro sujetos a los que les facilitaron las encuestas. En nuestro estudio nos centraremos en los resultados de las encuestas y estudios de recepción de los dos elementos seleccionados: la fachada del Ayuntamiento de Pamplona y el Jardín Bajo del Alcázar de los Reyes Cristianos de Córdoba que realizaron los sujetos (S 7), (S 8), (S 10) y (S 13).

La evaluación se dividió en tres fases:

a. Sesión informativa. Se informó a los participantes sobre el proyecto OPERA y los objetivos, las fases y el protocolo del estudio en el que iban a participar.

b. Los investigadores facilitaron la encuesta previa y la general del perfil turismo a los cuatro sujetos seleccionados en nuestro grupo.

c. Evaluación cuestionarios. Se les hizo los cuestionarios de cada uno de los materiales seleccionados. Cada estudio se compone de tres apartados: (1) cuestionario previo, que busca medir las variables sociodemográficas de los participantes (tipo y grado de discapacidad, educación, profesión y cuestiones generales relacionadas con el consumo cultural y los hábitos de uso de nuevas tecnologías de los participantes); (2) visionado o escucha de los segmentos seleccionados para el estudio; y (3) cuestionario de evaluación. El cuestionario de evaluación está formado por preguntas de respuesta cerrada y consta asimismo de tres apartados, según el tipo de variable independiente que se pretende medir:

1. Opinión y puntuación: este apartado se compone de preguntas de valoración general del recurso y específicas de la extensión y el grado de dificultad léxica y sintáctica percibidas por el participante que emplean la escala de Likert.

2. Recuerdo: este apartado se compone de preguntas de respuesta cerrada de respuesta única cuyo objetivo es comprobar la cualidad de ser recordado del segmento evaluado.

3. Comprensión: este apartado se compone de preguntas de respuesta cerrada de respuesta única cuyo objetivo es evaluar un aspecto concreto del recurso correspondiente a la variable dependiente de tipo lingüístico, textual, traductológico y técnico aislada en la primera fase del diseño. 


\section{Resultados}

\subsection{Resultados cuestionario general}

Con las respuestas de la encuesta previa pudimos extraer las siguientes conclusiones:

1. De los cuatro sujetos tres son mujeres (S 7) (S 8) y (S 13) y el (S 10) es varón.

2. Los cuatro sujetos están en un rango de edad (entre 40 y 60 años).

3. Dos sujetos (S 7) y (S 8) tienen estudios universitarios, y los otros dos (S 10) y (S 13) tienen el Bachillerato. Es decir, todos tienen un nivel académico alto.

4. Tres sujetos tienen una deficiencia visual grave (A 7) (S 19) y (S 13) y el (S 8) tienen una ceguera total. Todas son congénitas y dos de los sujetos (S 8) y (S 13) la han adquirido en los últimos 5-10 años.

5. Todos realizan actividades culturales: visitas al patrimonio cultural, a museos y a exposiciones. Y están de acuerdo en que haya entornos y contenidos adaptados.

\subsection{Resultados cuestionario turismo}

Todos hacen turismo con frecuencia y suelen hacerlo con amigos o familiares y el (S 13) además en grupos organizados.

1. Todos están de acuerdo en que visitarían más el patrimonio histórico y natural si fuera más accesible.

2. Todos esperan aprender y divertirse cuando hacen turismo.

3. Lo que más valoran de una visita accesible es la accesibilidad del entorno, la información que los rodea y también la actitud de los profesionales que trabajan allí.

4. Todos utilizan recursos de accesibilidad y entienden que las nuevas tecnologías facilitan el acceso a las personas ciegas a los espacios y al conocimiento.

5. Dos de los sujetos (S 8) y (S 13) prefieren visitas guiadas con exploración táctil mientras que el (S 7) prefiere una visita guiada con $A D$ y el (S 10) una visita autónoma con AD; y todos están de acuerdo en que una exploración táctil es muy útil para ellos y siempre mejor hacerla sin guantes.

6. Tres de los sujetes encuestados (S 7), (S 8) y (S 13) prefieren recibir la información en audio y el (S 10) prefiere braille y en ocasiones audio también y todos coinciden en que prefieren una maqueta seccionada para poder comprender la organización y distribución de los espacios.

7. En cuanto a la información que debe recoger una audioguía todos insisten en que debe ofrecer: una presentación del lugar del patrimonio histórico y cultural en el que se hallan, audiodescripción del entorno físico que rodea al visitante, indicaciones para realizar la visita, instrucciones para la exploración táctil, audiodescripción de los expositivos e interpretación y explicación de los expositivos.

8. Para descargarse la información los sujetos (S 7) y (S 10) prefieren su propio dispositivo mientras que los sujetos (S 8) y (S 13) prefieren el del lugar del patrimonio cultural que están visitando. Cuando usan su móvil todos coinciden en que prefieren descargarse la información en casa desde el sitio web. 
9. Dos de los sujetos encuestados (S 7) y (S 13) contestan que los responsables de accesibilidad en el patrimonio artístico no entienden las necesidades de las personas ciegas. El (S 8) no lo sabe y el (S 10) dice que sólo los entienden a veces.

10. El (S 8) y el (S 13) creen que siempre debe haber personas con diversidad funcional visual en los equipos de desarrollo y adaptación de los contenidos específicos para ellos, sin embargo, los (S 7) y (S 10) consideran que solo a veces es necesario que estén.

\subsection{Resultados cuestionarios específicos elementos seleccionados}

\section{- Ejemplo I. Fachada del Ayuntamiento de Pamplona}

1. Los cuatro sujetos encuestados valoran la AD como aceptable.

2. El (S 10) valora el lenguaje de la $A D$ como demasiado fácil, mientras que los otros tres lo valoran como fácil.

3. Tres sujetos consideran que la cantidad de información de la AD es adecuada, sin embargo el (S 10) la considera algo escasa.

4. La descripción de la fachada como un reloj de pared ha ayudado mucho a imaginársela al (S 7) y al (S 8) y bastante a los (S 10) y (S 13).

5. La comparación de la fachada como un mueble de cajones de piedra gris, sin embargo, ha ayudado un poco a entender la AD para los (S 10) y (S 13), al (S7) sí le ha ayudado mucho para poder imaginársela y al (S 8) le ha ayudado bastante.

6. La comparación de una de las figuras con Juana de Arco ha ayudado un poco a los cuatros sujetos a imaginársela.

7. En cuanto al estilo de la fachada el (S 7) dice que no lo sabe, el (S 8) dice que muy recargado y barroco, y los (S 10) y (S 13) dicen que muy sencillo.

8. En cuanto a los elementos arquitectónicos que recuerdan, los (S 7) y (S 13) recuerdan varios arcos, el (S 8) dice que no lo sabe y el (S 10) dice que hay figuras femeninas.

\section{- Ejemplo II. Jardín Bajo de los Alcázares de los Reyes Cristianos en Córdoba}

1. Al (S 7) no le ha gustado nada la $A D$, al (S 8) le ha gustado bastante y a los (S 10) y (S 13) le ha gustado un poco.

2. Tres sujetos (S 7), (S 10) y (S 13) opinan que la información que incluye la AD es escasa, sin embargo para el (S 8) es suficiente.

3. Los cuatro opinan que las oraciones son adecuadas.

4. Tres de los sujetos (S 7), (S 10) y (S 13) no se han podido imaginar el jardín, sin embargo, el (S 8) ha comprendido la $A D$ y se imagina el jardín.

5. En cuanto a la superficie del jardín el (S 7) y el (S 10) consideran que es extensa, el (S 13) muy extensa y el (S 8) pequeña. 
6. Tres sujetos se imaginan el jardín con flores de vivos colores pero con pocos árboles y otro sujeto (S 13) se lo imagina con muchos árboles y flores de colores vivos.

7. Para los sujetos (S 7), (S 8) y (S 13) toda la información es pertinente, pero el (S 10) habría preferido información sobre otros aspectos como los orígenes del jardín, el sistema de riego o los tipos de árboles.

8. En cuanto a la información que han echado en falta, el (S 7) dice que la fauna que hay en el jardín y la situación de los restos arqueológicos, el (S 8) dice que no le falta información, el (S 10) requiere otro tipo de información y el (S 13) la situación de los restos arqueológicos.

9. El (S 7) y el (S 13) coinciden en que la AD se debería ampliar con más información histórica, más información sobre las características de la fuente, los estanques y las acequias, también más información sobre la vegetación del jardín y sobre la comunicación de los jardines con el castillo. El (S 8) demanda otro tipo de información y el (S 10) se queda con más información sobre las características de la fuente y sobre la vegetación del jardín.

\section{Discusión de los resultados}

De los resultados expuestos en el apartado anterior hemos de señalar que los cuatros sujetos coinciden en que lo que más valoran de una visita accesible es la accesibilidad del entorno, la información que los rodea y también la actitud de los profesionales que trabajan allí. Además, todos utilizan recursos de accesibilidad y entienden que las nuevas tecnologías facilitan el acceso a las personas ciegas a los espacios y al conocimiento. De los recursos para acceder a la información del patrimonio histórico los cuatros sujetos han seleccionado todos los que la encuesta les ofrecía: audiodescripción, impresión en termorrelieve, sonidos, olores y texturas, alimentando así en los profesionales la necesidad de crear todos estos recursos. Los encuestados también entienden que la información en audio les facilita mucho más el conocimiento del patrimonio, de manera que nos afirma en nuestra trayectoria como creadores de AD y además como formadores de profesionales de la AD. Todos son usuarios de teléfonos inteligentes y en este sentido prefieren bajarse la información del lugar que van a visitar desde la web propia del lugar. Coinciden además en que casi nunca se entienden las necesidades de accesibilidad de este colectivo y apuntan que sería bueno que en los equipos de trabajo sobre accesibilidad hubiera personas ciegas que prestaran su apoyo.

Entre los visitantes con diversidad funcional visual hay una gran variedad de opiniones: algunas personas requieren y disfrutan de una audiodescripción muy detallada, mientras que otras dan menos importancia a la audiodescripción y prefieren más información contextual. En este sentido, entendemos que la AD del patio necesita en general de muchas mejoras. En general los sujetos evaluados opinan que la información ofrecida en la $A D$ es escasa. $Y$ en cuanto a si les ha gustado o no, hay variedad de opiniones.

De hecho tres de los sujetos no se han podido imaginar cómo es el jardín aunque coinciden en que las oraciones empleadas para describirlo son adecuadas. De lo que podemos inferir, lógicamente que la información es escasa. 
Mientras que la AD de la fachada del Ayuntamiento de Pamplona, sin embargo, ha sido mejor entendida y, por tanto, mejor valorada. De hecho tres de ellos consideran que la información ofrecida es adecuada. Es interesante analizar las comparaciones que se aportan en la AD de la fachada, cómo la más común, la del "reloj de pared" es la más aceptada y apropiada para entenderla, mientras que las otras no lo son tanto. Sobre todo la de la figura que se compara con Juana de Arco, posiblemente a la persona ciega no le diga nada, si no conoce la historia de la heroína. También parece que no han ayudado mucho las descripciones de la fachada, pues tanto el estilo como la distribución de los pisos que la conforman deberían haberse explicado de manera más clara y sencilla.

En conclusión, cuando se aborda el desarrollo de una guía audiodescriptiva u otra clase de recurso de accesibilidad turística es fundamental tener presente que, de la misma forma que los visitantes sin discapacidad no conforman un grupo homogéneo, los visitantes con diversidad funcional visual presentan características muy distintas relacionadas con el tipo y grado de discapacidad, pero también con su conocimiento previo, hábitos culturales, edad y, cómo no, con sus gustos personales.

\section{Conclusiones}

En este artículo hemos presentado los resultados de la evaluación formativa de dos de los contenidos creados para la plataforma digital PRA2 desarrollados para el proyecto OPERA con el fin de mejorar la calidad de los materiales creados ex profeso para la plataforma y los seleccionados de otras instituciones que han cedido los mismos para su evaluación por los usuarios de la plataforma. Además hemos recogido los resultados de una evaluación formativa en la que el rasgo predominante del perfil de los receptores era su diversidad funcional visual. Con ello pretendemos aportar un pequeño grano de arena al aumento de los estudios de visitantes del patrimonio artístico, en nuestro caso en concreto, de visitantes del patrimonio con diversidad funcional visual. Su colaboración ha proporcionado al estudio un nada despreciable ejercicio valorativo a modo de encargo traductor real. Si bien el escaso número de sujetos no nos ha permitido obtener resultados definitivos, sí nos ha ofrecido informaciones valiosas acerca del perfil de visitantes con diversidad funcional visual que nos han servido para la elaboración de contenidos accesibles que irán alojados en la plataforma digital, es decir, nos han permitido formarnos una imagen de los posibles receptores prototipos meta de las traducciones accesibles.

Para la evaluación de los contenidos, hemos elegido dos textos: por un lado la fachada del Ayuntamiento de Pamplona, y, por otro, el Jardín Bajo de los Alcázares Cristianos de Córdoba y, aunque los resultados no son representativos, sí han permitido observar que se ha logrado la funcionalidad del texto y la terminología empleada ha sido adecuada. No obstante, también ha sido posible observar que los sujetos no están de acuerdo en todas las cuestiones y, de este modo, han disentido, por ejemplo, en la calidad de la audiodescripciones, así como en la información alojada en ellas.

Gracias a nuestros análisis y presentación de los resultados de la evaluación formativa hemos pretendido engrosar la pequeña lista de los estudios dedicados a los turistas y visitantes del patrimonio histórico y 
cultural con unas necesidades específicas. Así pues, entendemos que el experto en accesibilidad turística deber seguir trabajando en esta dirección para facilitar el acceso a la cultura y cualquier espacio a las personas con diversidad funcional, así como dar un paso más hacia una formación de traductores e intérpretes expertos en accesibilidad turística. 


\section{Referencias bibliográficas}

AENOR (2012): Norma UNE 153010: Subtitulado para personas sordas y personas con discapacidad auditiva. Madrid: AENOR.

AENOR (2005): Norma UNE 153020: Audiodescripción para personas con discapacidad visual. Requisitos para la audiodescripción y elaboración de audioguías. Madrid: AENOR.

Fryer, L. y Freeman, J. (2012): "Presence in those with and without sight: audio description and its potential for virtual reality applications". Journal of Cyber Therapy \& Rehabilitation, 5 (1): 15-23.

Iglesias, E. et al.: (2015): "Cross-fertilization between reception studies in audio description and interpreting quality assessment: the role of the narrator's voice”, en Díaz, J. et al. (eds.). Audiovisual Translation: Taking Stock. Amsterdam: Rodopi.

Jiménez, C. et al. (2012): "Museos para todos. La traducción e interpretación para entornos multimodales como herramienta de accesibilidad universal”. MonTI. Monografías de Traducción e Interpretación, 4: 349-383.

Mazur, I. y Kruger, J. L. (eds.) (2012): "Pear Stories and Audio Description: Language, Perception and Cognition across Cultures". Perspectives Studies in Translatology, 20 (1): 23-32.

Navarrete, J. (1997): “Sistema AUDESC: el arte de hablar en imágenes”. Integración, 23: 70-75.

Peli, E. et al. (1996): "Evaluating visual information provided by audio description". Journal of Visual Impairment and Blindness, 90 (5): 378-385.

Pettitt, B. et al. (1996): "AUDETEL: enhancing telesight for visually impaired people”. British Journal of Visual Impairment, 14 (2): 48-52.

Soler, S. y Chica, A. J. (2014): "Museos para todos: evaluación de una guía audiodescriptiva para personas con discapacidad visual en el museo de ciencias". Revista Española de Discapacidad, 2 (2): 145-167. 